\title{
Evaluation Method and Empirical Research of Regional Water-Energy- Food Synergetic Development
}

\author{
Guiqin Jiang ${ }^{1, \mathrm{a}}$, Maihuan $\mathrm{Zhao}^{1}$, Qiong $\mathrm{Su}^{1}$ and Yiqing $\mathrm{He}^{1}$ \\ ${ }^{1}$ Yellow River Engineering Consulting Co.,Ltd., Henan 450003, China
}

\begin{abstract}
Regional water-energy-food (WEF) synergetic development evaluation is an important prerequisite for promoting regional cooperative security development effectively. Under the framework of regional WEF nexus system, the concept of regional WEF synergetic development was proposed based on the synergetic theory, and evaluation index system was constructed from the aspects of function, structure and efficiency. The development degree was evaluated by measuring the gap between the existing state and the ideal state based on the distance synergy method. Considering the synergy relationship between each subsystem, the grey correlation analysis method was used to determine the pull factors between each subsystem, and then the synergy degree was calculated. The synergetic development degree was calculated by integrating development degree and synergy degree. This method was used to analyse the WEF synergy development degree of Ningxia Hui Autonomous Region in China from 2001 to 2015. The results show that the WEF synergetic development of Ningxia was increasing but still at a low level, food subsystem had significant pull function to the water and energy subsystems. While improving the development degree of each subsystem, Ningxia needs to strengthen the collaborative development among the subsystems to improve the synergetic development of regional water, energy and food.
\end{abstract}

\section{Introduction}

Water, energy and food are not only the necessary resources for human survival, but also the "slow variables" or "defect" to regional sustainable development system, for the regional sustainable development is restricted by the integrated changes of Water, energy and food. Enhancing the system resilience by accelerating the WEF synergetic development effectively and promoting the efficiency of integration utilization of WEF can response to threats from external impacts, which is helpful for achieving the regional sustainable development [1]. Resource security is the fundamental guarantee of regional sustainable development, and regional water-energy-food (WEF) synergetic development evaluation is an important prerequisite for promoting regional cooperative security development effectively.

There are plenty of the national and overseas researches on single resource among Water, energy and food. However, the water-energy-food synergetic development is seldom studied, which becomes the one of the difficult problems for current research. Some researchers study the WEF synergetic development from aspect of system science, the achievements of the researches are divided into two categories: one is qualitative analysis, where the nexuses among Water, energy and food are focused on to analyse one resource changing impacts on the other two resources development or whole system sustainable development, then the suggestions are proposed from the macro policies [2-3]; the other one is quantitative analysis, the digitized results are obtained by processing related index data with mathematical model. There are two kinds of quantitative analysis includes thoughts: one is that the system synergy extent is reflected by testing the synergetic effects from synergetic factors, for instance, Peng Deng[4], Bo Bi[5], etc. implemented the couplingcoordinating model to research the couplingcoordinating evolution features of WEF system; the other one is that evaluating the system synergy degree by measuring the gap between the current status and ideal status, or estimating the progress extent comparing with the basic status, such as Shaoming Peng[6], etc., who proposed the computation method of basin WEF comprehensive operation based on the distance synergy thought, and presented the WEF synergetic optimal distribution in Yellow River Basin with the optimal objective of comprehensive regulation.

Both the qualitative analysis and the quantitative analysis are used in this study, the regional WEF synergy development concept was proposed based on the qualitative analysis of WEF system construction; evaluation index system of the WEF synergy development was established, which was integrated with grey correlation method and distance synergy method to put forward the quantitative evaluation method of regional WEF synergy development; finally, the Ningxia Hui Autonomous Region is as study case to empirical analysis.

\footnotetext{
${ }^{a}$ Corresponding author: jiangguiqin1@163.com
} 


\section{Regional WEF synergy development}

\subsection{System construction}

Water, energy and food, each of them are highly concerned as the significant problem in the sustainable development research field, however, the integration of these three resources were attached the enough importance. Until 2011, the studies on nexus of Water, energy and food (WEF-Nexus) were becoming the hot point of research. In Nov. 2011, the international conference for discussing WEF-Nexus was held by The German Federal Government in Bonn, in order to explore way of green economy development. The conference pointed that, the production and consumption of Water, energy and food are impacted by global climate and social changes, the nexus among these three resources are complex, especially, the trade-off and potential conflicts generally exist within the process of production, consumption, and management, so that any single resource strategy can lead to the unpredictable severe consequence [7].

Regional WEF system is the resources system with a complex structure and various functions. The WEF system can be divided into water subsystem, energy subsystem and food subsystem, and these three system are interacted and impacted each other, which forms an intricate nexus. This WEF-nexus is mainly reflected in that: irrigation is requested by food production, meanwhile the virtual water included in other production factors is integrated into food production; the energy input is demanded in processes of food production which including irrigation, fertilization, transportation, processing, and so on; food can be used as production source of energy, such as biofuel; water input is needed in energy producing and processing, also the energy is input into water exploring, processing, supplying, purifying and other processes. At the same time, the WEF-nexus is influenced by climate change, population growth, economy development, environmental pressures, and other factors outside system. The figure of regional WEF-nexus conceptual framework is shown in figure 1.

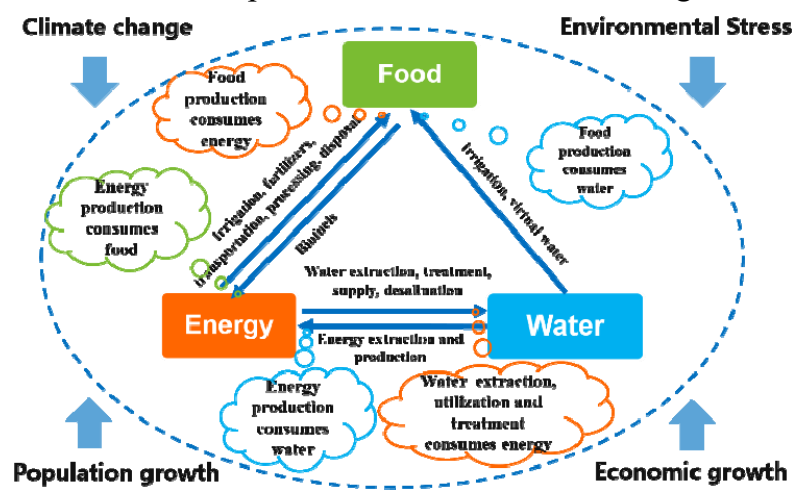

Figure 1. Regional WEF-nexus conceptual framework.

\subsection{System synergetic development}

The word synergy comes from ancient Greek, which can also be addressed as consonance, synchronization, harmony, coordination, cooperation or collaboration, which is belonged into basic category of synergetic. The meaning of synergy is that the process or capacity to achieve one objective by two and more than two different resources or individuals. Synergy development is presented as a virtuous circle process with every subsystem adapting, coordinating, cooperating and promoting each other in the system [8]. The internal and external consumption can be decreased obviously by synergy development which also can motivate the enthusiasm and generate the complementary effect, furthermore, the efficiency of resources utilization and efficiency of production can be improved; the " $1+1>2$ " effect can be stimulated through the aggregating integrity, comprehensiveness and endogenous effect under the prompting of synergy development [9-10].

Regional WEF synergy development is refer to a process where WEF can achieve balance between supply and demand, structure rationalization, efficient operation and friendly environment after interaction and influence to each other within subsystem or among subsystems under the regional WEF nexus framework.

\section{Methodology of WEF synergetic development evaluation method}

\subsection{Evaluation index system construction}

In terms of a system, balance of every main factors, reasonable structure, effective operation, ordered exchange of energy and substance play a significant role and can help system exist longer, so that the system can be more developed. According to the construction and synergism of regional WEF system, and following the scientificity, practicability, dynamics, data availability, and other principles, the indicator system of regional WEF synergy development was built from aspects of functions, structure, efficiency and symbiosis in water, energy and food these three system. The 22 indicators are shown in table 1.

Table 1. Evaluation index system of WEF synergetic development.

\begin{tabular}{lllll}
\hline Subsystem & Feature & Evaluation index & Unit & Index type \\
\hline 1. Water system & 1.1 Function & 1.1.1water resource per capita & $\mathrm{m}^{3} /$ capita & + \\
& & 1.1 .2 water use per capita & $\mathrm{m}^{3} /$ capita & -
\end{tabular}




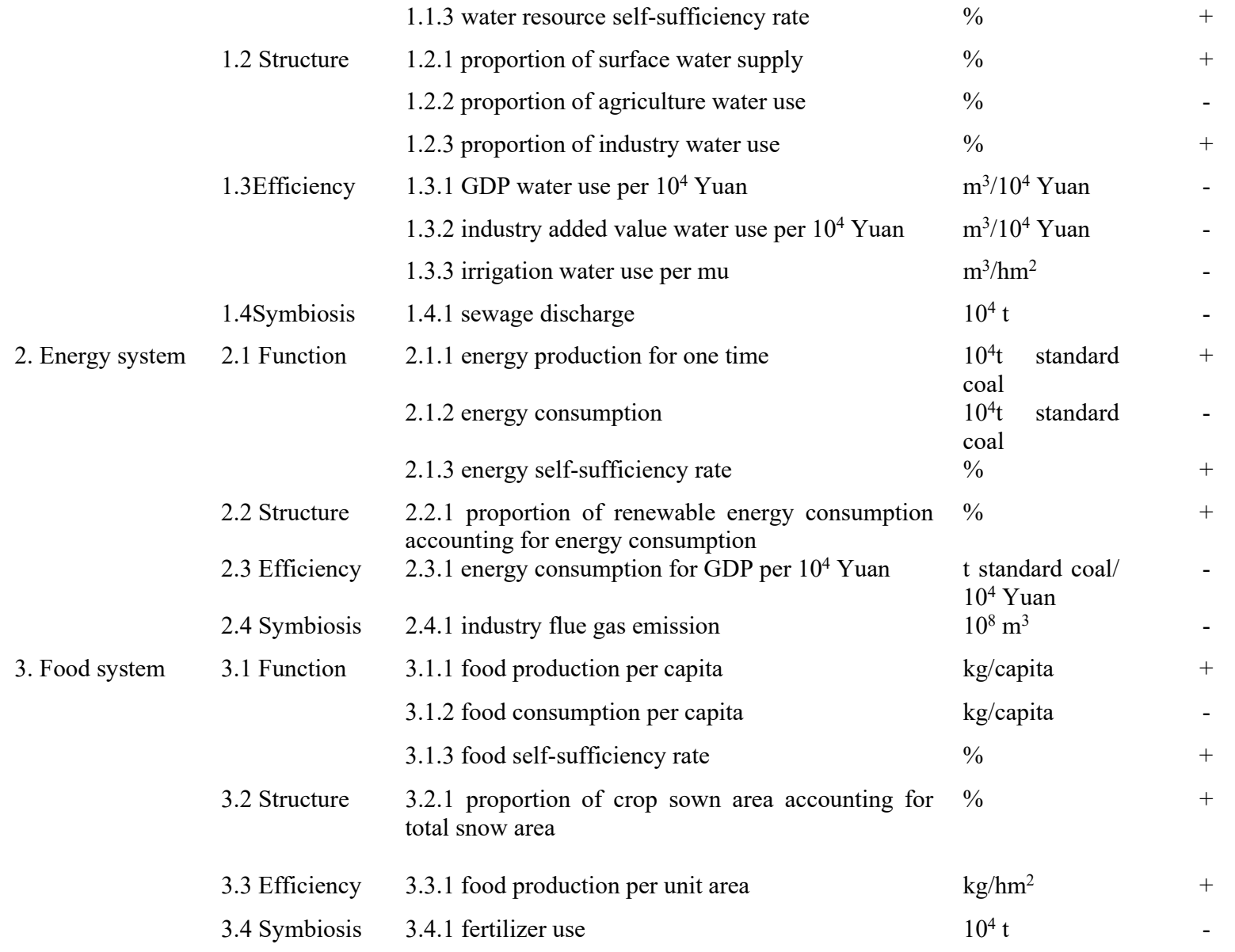

Note: "+" indicates that the bigger the better while "-" refers that the smaller the better.

$$
a_{i j t}=\left(x_{i j t}-\min x_{i j}\right) /\left(\max x_{i j}-\min x_{i j}\right)
$$

\subsection{Calculation of synergetic development extent}

The theory of distance synergy method is that the system related state is measured by Euclidean distance between system practical state and system ideal state, however, the difference of pulling effect among subsystems is not considered. Haidong $\mathrm{Li}$, etc. improve that model with TOPSIS thought and grey correlation theory aiming at those insufficient. In this research, the improved model is used to evaluate the regional WEF synergy development extent. First, the indicators of each subsystem were normalized. Then, the optimal vector and the worst vector is input into model, the development extents of each subsystem were measured with distance ratio, and the comprehensive development degree of whole WEF system is obtained through the geometric mean of each subsystem development degree. After that, the pulling factors were determined using grey correlation of subsystems in order to get the synergy degree of each subsystem and comprehensive synergy degree of whole WEF system. Finally, the synergy development extent can be obtained by computing the geometric mean between development degree and synergy degree. The details are shown below:

(1) Normalize evaluation indicator.

For the number $\mathrm{j}$ index of I subsystem in t period, the normalization method shown as following:

The bigger the better indicator:
The smaller the better indicator:

$$
a_{i j t}=\left(\max x_{i j}-x_{i j t}\right) /\left(\max x_{i j}-\min x_{i j}\right)
$$

Where: is the indicator after normalization, and stand for the maximum and minimum of $\mathrm{j}$ index in $\mathrm{i}$ subsystem during the studied period, respectively.

(2) Determine the positive ideal points and negative ideal points of each subsystems

Positive ideal point: $A^{+}=\left(a_{i 1}^{+}, a_{i 2}^{+}, \ldots, a_{i m}^{+}\right)$is the set of the optimal values.

Negative ideal point: $A^{-}=\left(a_{i 1}^{-}, a_{i 2}^{-}, \ldots, a_{i m}^{-}\right)$is the set of the worst values.

(3) Compute the development degree of each subsystem and whole WEF system.

First, compute the distances $D_{i t}^{+}$between every subsystem and positive ideal point, and distances $D_{i t}^{-}$between every subsystem and negative ideal point:

$$
\begin{aligned}
& D_{i t}^{+}=\sqrt{\sum_{j}\left(a_{i j}^{+}-a_{i j t}\right)^{2}} \\
& D_{i t}^{-}=\sqrt{\sum_{j}\left(a_{i j}^{-}-a_{i j t}\right)^{2}}
\end{aligned}
$$

Where, $a_{i j}^{+} 、 a_{i j}^{-}$refer to the number $\mathrm{j}$ value of vector and vector, respectively.

Then, calculate the development degrees of subsystems:

$$
d_{i t}=\frac{D_{i t}^{-}}{D_{i t}^{-}+D_{i t}^{+}}
$$


Where, $d_{i t}$ is determine in range from 0 to 1 , the closer to 1 the higher development degree, on the contrary, the closer to 0 the lower development degree.

Finally, compute the comprehensive development degree of the whole WEF system ${ }^{d}$ :

$$
d_{t}=\sqrt[3]{\prod_{i}^{3} d_{i t}}
$$

(4) Compute the correlation factors among subsystems and decide the pulling factor.

The development degree values of subsystems is implemented as original data series, the grey comprehensive correlation degree $\alpha_{i j}$ is computed. If the $j$ subsystem curve is upon the $i$ subsystem curve, the $j$ subsystem develops ahead of $i$ subsystem, so the pulling effect is less than 1 to $i$ subsystem by $j$ subsystem, then set the pulling factor between $j$ subsystem and $i$ subsystem as that $\beta_{i j}=\alpha_{i j}$. If the $\mathrm{j}$ subsystem curve is under the $i$ subsystem curve, the $\mathrm{j}$ subsystem develops after of $i$ subsystem, so the pulling effect is more than 1 to $i$ subsystem by $\mathrm{j}$ subsystem, then set the pulling factor between $j$ subsystem and $i$ subsystem as that $\beta_{i j}=1 / \alpha_{i j}$. The pulling factor, $i$ subsystem act on itself, is 1 . The calculation steps of grey correlation is are shown below:

First, calculate the absolute correlation: initialize the original set $X_{i}=\left(d_{i 1}, d_{i 2}, \ldots, d_{i t}\right)$, compute the value equal to 0 .

$$
\begin{array}{r}
X_{i}^{0}=\left(d_{i 1}-d_{i 1}, d_{i 2}-d_{i 1}, \ldots, d_{i t}-d_{i 1}\right)=\left(d_{i 1}^{0}, d_{i 2}^{0}, \ldots, d_{i t}^{0}\right) \\
\text { Set } \\
\qquad\left|z_{i}\right|=\left|\sum_{k=2}^{t-1} d_{i t}^{0}+\frac{1}{2} d_{i t}^{0}\right| \\
\left|z_{i}-z_{j}\right|=\left|\sum_{k=2}^{t-1}\left(d_{i t}^{0}-d_{j t}^{0}\right)+\frac{1}{2}\left(d_{i t}^{0}-d_{j t}^{0}\right)\right|
\end{array}
$$

Then the absolute correlations of subsystem $i$ and $j$ are:

$$
\mu_{i j}=\frac{1+\left|z_{i}\right|+\left|z_{j}\right|}{1+\left|z_{i}\right|+\left|z_{j}\right|+\left|z_{i}-z_{j}\right|}
$$

As following, calculate the relatively correlation, change the original series in absolute correlation to $X_{i}=\left(\frac{d_{i 1}}{d_{i 1}}, \frac{d_{i 2}}{d_{i 1}}, \ldots, \frac{d_{i t}}{d_{i 1}}\right)$ which shows the correlation degree of change ratio of subsystem $i$ and $j$, of subsystem $i$ and $j$ with the same method.

Finally, calculate the comprehensive correlation $\alpha_{i j}=\theta \mu_{i j}+(1-\theta) v_{i j}$, the correlations among subsystems can be represented comprehensively by grey correlation.

(5) Compute the synergy degree of subsystems and entire WEF system.

First, calculate the ideal development degree of subsystem $^{d_{i t}^{\prime}}$ :

$$
d_{i t}^{\prime}=\sqrt[k]{\prod_{j=1}^{k} \beta_{i j} d_{j t}}
$$

Then, calculate the synergy degree of subsystem ${ }^{S_{i t}}$ : $s_{i t}=\frac{\left|d_{i t}\right|}{\left|d_{i t}\right|+\left|d_{i t}-d_{i t}^{\prime}\right|}$

Finally, compute the comprehensive synergy degree of entire WEF system ${ }^{s_{t}}$ :

$$
S_{t}=\sqrt[k]{\prod_{i=1}^{k} S_{i t}}
$$

(6) Calculate the comprehensive synergy development degree of entire WEF system.

The comprehensive synergy development degree of entire WEF system $C_{t}$ :

$$
C_{t}=\sqrt{s_{t} d_{t}}
$$

\section{Case study}

\subsection{Profile of study area}

The Ningxia Hui Autonomous Region is researched as study case in order to test the practicality of the evaluation of regional WEF synergy development. Ningxia is located in northwest inland area of China, the total area is $6.64 \times 10^{4} \mathrm{~km}^{2}$, the population is $6.679 \times 10^{6}$ people and GDP is $2927.96 \times 10^{8}$ Yuan. The water, energy and food situation is addressed as follow:

(1)Water resource overview. The water resource is shortage in Ningxia, so it has highly dependence on Yellow River. The regional water resource available per capita (include water from Yellow River) only accounts for $1 / 3$ national average level, especially outside the range of Yellow River Pumping Project in centralsouthern Ningxia, where the water resources per capita is much less. In 2015, the GDP water use per 104 Yuan of whole region is 2.7 times than national average, the general agricultural irrigation method is field flooding irrigation, the regional average irrigation water utilization coefficient is 0.501 , which is lower than the national average level 0.536; agricultural water use take up $88.1 \%$ of regional water use, that is much higher than national average proportion $63.1 \%$. Since the water of Yellow River comes less while regional water demand growths up, the conflict between water demand and water supply will be severer in Ningxia in future.

(2) Energy resources overview. The energy resources are abundance in Ningxia, which has advantages of coalbased multi-energy complementary and comprehensive development. The added value of energy industry accounts for more than $40 \%$ of the added value of regional industry in Ningxia, so that it becomes the most significant energy processing and strategic reserving base, and play a very important role in national energy strategy. The regional explored coal storage is 40.1 million $t$ in Ningxia, the potential resource storage is 147.1 billion $t$. In 2015 , the regional coal production is 79.76 million $\mathrm{t}$, power capacity is 31.54 billion $\mathrm{kW}$, the power generation is 136.8 billion $\mathrm{kW} \cdot \mathrm{h}$, moreover, the power capacity per capita and power generation per capita is ranked first in China, therefore, Ningxia is one 
of the most significant energy base according to the "West-East power transmission" strategy. In addition, there are plenty of wind energy, solar energy, and many other new energy resources in Ningxia, where the new energy development level keeps advanced in the whole country.

(3)Food overview. The agricultural area was 1.59 million hm2 in Ningxia in 2015, the food production was 3.75 billion $t$, in detail, the wheat production was 393 thousand $t$, paddy production was 705 thousand $t$, corn production was 2.23 million $\mathrm{t}$, and potato production was 393 thousand $t$. The regional annual food supply was 4.38 million $t$ (where the around 700 thousand $t$ food was purchased by other provinces), the food demand was 4.25 million $\mathrm{t}$ (where local consumption was 3.59 million $t$, food soled to outside the region was 655 thousand, the export was 2 thousand $t$ ), so the food supply was generally matched the food demand. Although there is a basic balance between food supply and food demand in Ningxia, the wheat and edible oil are in high external dependence, and there exist an obvious conflict on planting structure of food varieties.

\subsection{Data sources}

In this research, the WEF synergy development states of Ningxia from 2001 to 2015 were analyzed, the data of function, structure, the efficiency of water subsystem were obtained from China Water Resources Bulletin (2001-2015), the data of function, structure, the efficiency of energy subsystem were found from China Energy Statistics Yearbook (2002-2016), the symbiosis indicators from water subsystem and energy subsystem (such as sewage discharge, industry flue gas emission) and food subsystem were referred from the China Statistics Yearbook (2002-2016).

\subsection{Results and discussion}

The results of comprehensive development degree and the development degree of water subsystem, energy subsystem and food subsystem are shown in figure 2 . Though the calculation grey correlation of subsystems, the full factors among each subsystem is illustrated in table 2. On the basis of above, the comprehensive synergy degree and synergy degrees of every subsystem from 2001 to 2015 are described in figure 3. Furthermore, the comprehensive synergy development degree and synergy development degrees of water subsystem, energy subsystem and food subsystem are demonstrated in figure 4.

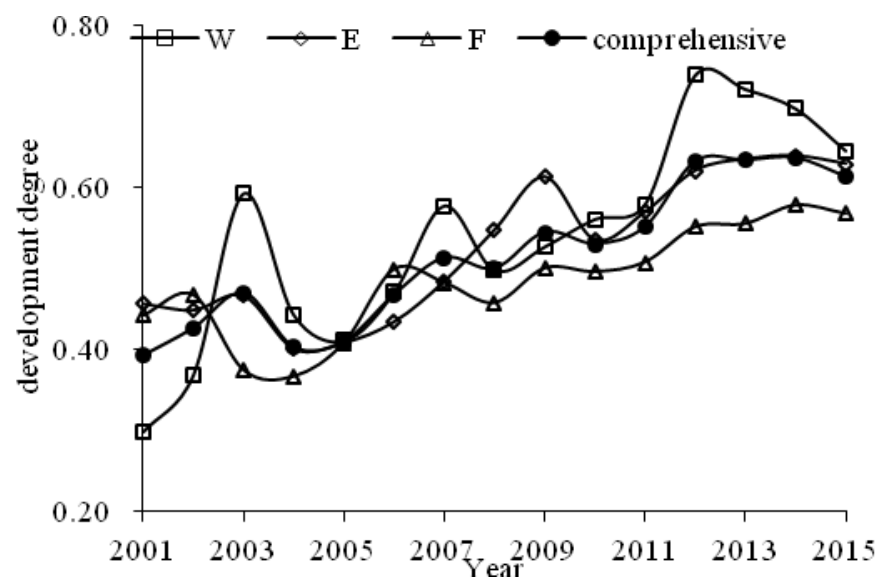

Figure 2. WEF subsystem development degree and comprehensive development degree in Ningxia.

Table 2. Pull factors among water subsystem, energy subsystem and food subsystem in Ningxia.

\begin{tabular}{cccc}
\hline pull factor & W & $\mathbf{E}$ & F \\
\hline W & 1.0000 & 1.1800 & 1.2615 \\
E & 0.9050 & 1.0000 & 1.0649 \\
F & 0.8518 & 0.9421 & 1.0000 \\
\hline
\end{tabular}




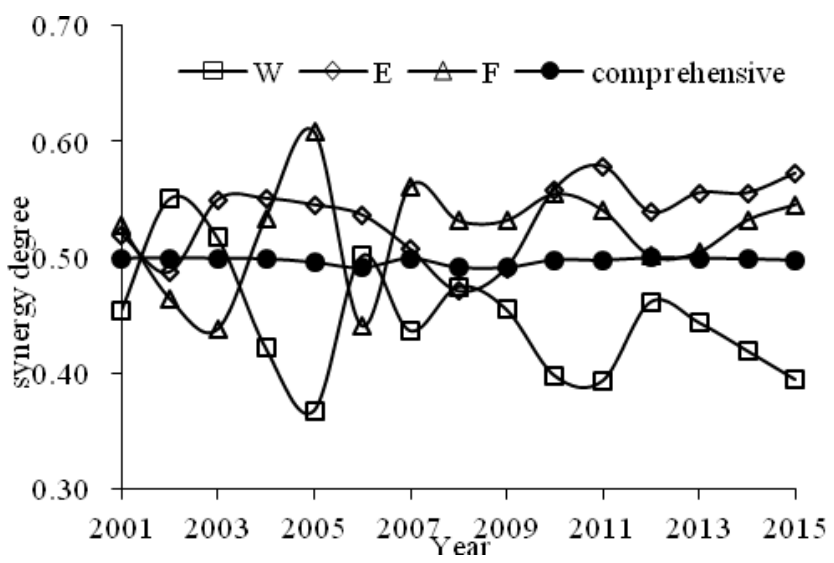

Figure 3. Comprehensive synergy degree and synergy degrees of every subsystem in Ningxia from 2001 to 2015.

According to results, the conclusions can be obtained as below:

(1) The comprehensive development of WEF system in Ningxia from 2001 to 2015 increases significantly (shown in figure 2). The water system development degree rise from 0.2983 in 2001 to 0.7383 in 2012, which arrived the peak, then generally decreased to 0.6451 in 2015, result in heavier precipitation in 2012, water resource self-sufficiency were higher, and irrigation quota per mu was smaller than other years; the energy subsystem development degree went to a fluctuating increasing trend, and reached the top in 2009, which was mainly impacted by rapidly increasing industry flue gas emission after 2010; the food subsystem development degree increased steadily.

(2) The food subsystem has an obvious pull effect on water subsystem and energy subsystem. The pull factors between food subsystem and water subsystem, food subsystem and energy subsystem were 1.2615 and 1.0649, respectively, which showed that water subsystem and energy subsystem development slower (table 2). From the figure 2, every subsystem development degrees were significant different among years, however, the water subsystem development degree was higher than energy subsystem development degree, and the food subsystem degree was lowest, this state was kept stable after 2010.

(3) The comprehensive synergy degrees of WEF system in Ningxia from 2001 to 2015 went stable, which stayed round 0.5 . From the aspect of subsystem synergy, the water subsystem synergy degree declined in fluctuation, energy subsystem synergy degree climbed slowly, and the food subsystem synergy degree went steady (figure 3). The difference between system development state and system ideal development state was reflected on synergy degree, which can demonstrate that there was a larger and larger gap between development degree and ideal development degree in water subsystem while the development state got closer to ideal state in energy subsystem. In conclusion, the food and energy need to be paid more attention, at the same time, the water subsystem also need to be developed in order to improve the comprehensive synergy of the entire system.
(4) Comprehensive development synergy degree of WEF system in Ningxia had a rising trend from 2001 to 2015 , but the value was small between 0.4 and 0.6 (figure 4). The development degree was lower than synergy degree from 2001 to 2007, which addresses that the relationships are reasonable among each subsystem, but the development degrees of subsystems were small; development degree were bigger than synergy degree gradually during 2008 to 2015 , which gradually was outperformed the coordination of system relations and the rational structure of the whole system. Above all, for the sake of improving the regional WEF comprehensive synergy development degree, the development degree of each subsystem need to be enhanced, meanwhile, the synergy development degrees among subsystems need to be improved as well.

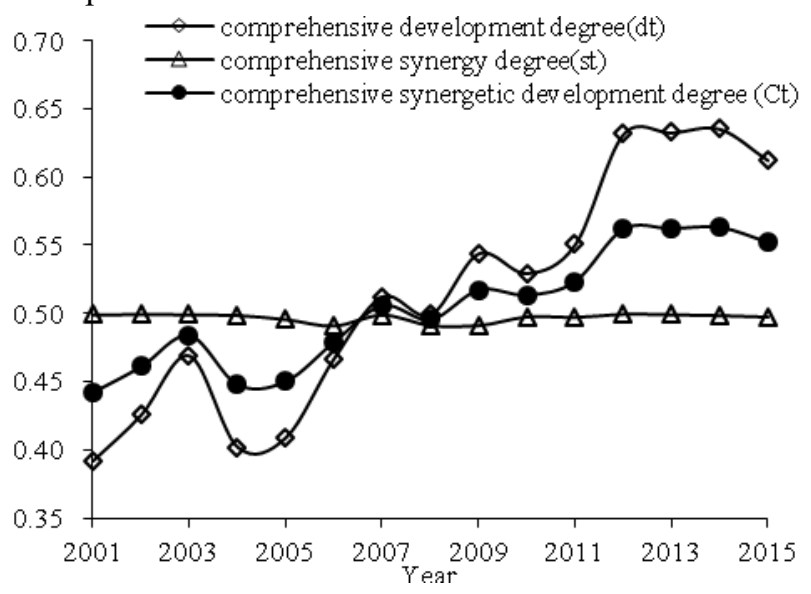

Figure 4. Comprehensive synergy development degree and synergy development degrees of water subsystem, energy subsystem and food subsystem in Ningxia from 2001 to 2015.

\section{Conclusions}

The concept of regional WEF synergy development was proposed based on the analysis of regional WEF system construction in this research; the indicator system of regional WEF synergy development was built from aspects of functions, structure, efficiency and symbiosis in water, energy and food these three system; the quantitative evaluation method of regional WEF synergy 
development was put forwards integrated with grey correlation method and distance synergy method; the Ningxia Hui Autonomous Region is researched as study case. The results shows that: the results of WEF development evaluation were identical to practice, so that the research method is operative and reasonable. However, the best value and the worst value of various evaluation indicators in different region and different stage change with diverse area and diverse development period, how to determine a universal best value and worst value is a problem need further researches.

\section{Acknowledgments}

The study is financially supported by the National Key Research and Development Program Project (Grant NO. 2017YFC0404604)

\section{References}

1. Guijun Li, Daohan Huang, Yulong Li, The Nexus of Water-Energy-Food: A New Niew of Regional Sustainable Development, Journal of Central University of Finance and Economics, 12, 76-90 (2016)

2. M.Gulati, I.Jacobs, A.Jooste, D.Naidoo and S.Fakir. The water-energy-food security nexus: Challenges and opportunities for food security in South Africa, Aquatic Procedia, 150-164 (2013)

3. Rae Zimmerman, Quanyan Zhu, Carolyn Dimitri, Promoting resilience for food, energy, and water interdependencies, Environ. Stud. Sci., 6, 50-61, (2016)

4. Peng Deng, Qing Chen, Dan Chen, etc., The evolutionary characteristics analysis of the coupling and coordination among water, energy and food: take Jiangsu Province as an example, Journal of Water Resource and Engineering, 6, 232-238 (2017)

5. Bo Bi, Dan Chen, Peng Deng, etc., The Evolutionary Characteristics Analysis of Coupling and Coordination of Regional Water-Energy-Food, China Rural Water and Hydropower, 2, $72-77$ (2018)

6. Shaoming Peng, Xiaokang Zheng, Yu Wang, etc., Water-Energy-Food Synergy Optimization of Yellow River Basin, Advances in Water Science, 5, 681-690 (2017)

7. Hoff H. Understanding the Nexus, Background Paper for the Bonn 2011 Conference: The Water Energy and Food Security Nexus (2011)

8. Haidong Li, Shuai Wang, Yang Liu, Regional Synergy Development Evaluation Method and Empirical Research Based on Grey Correlation Theory and Distance Synergy Model, Systems Engineering - Theory \& Practice, 7, 1749-1755 (2014)

9. Zhiping Du, Dong Mu, A Study on DEA Evaluation for Synergetic Development of System, Mathematics in Practice and Theory, 2, 75-81 (2005)
10. Dong $\mathrm{Mu}$, Zhiping Du, Study on the Evaluation of Cooperation Development of Resource Region, China Soft Science, 5, 106-113 (2005) 